\title{
A Robust Realtime Surveillance System ${ }^{\star}$
}

\author{
Byung-Joo Kim ${ }^{1}$, Chang-Bum Lee ${ }^{2}$, and Il-Kon Kim ${ }^{3}$ \\ ${ }^{1}$ Youngsan University Dept. of Information and Network Engineering, Korea \\ bjkim@ysu.ac.kr \\ ${ }^{2}$ Youngsan University Dept. of Information and Network Engineering, Korea \\ cblee@ysu.ac.kr \\ ${ }^{3}$ Kyungpook National University Dept. of Computer Science, Korea \\ ikkim@knu.ac.kr
}

\begin{abstract}
This paper describes a feature extraction method for realtime surveillance. Eigenspace models are a convenient way to represent set of images with widespread applications. In the traditional approach to calculate these eigenspace models, known as batch PCA method, model must capture all the images needed to build the internal representation. This approach has some drawbacks. Since the entire set of images is necessary, it is impossible to make the model build an internal representation while exploring a new person. Updating of the existing eigenspace is only possible when all the images must be kept in order to update the eigenspace, requiring a lot of storage capability. In this paper we propose a method that allows for incremental eigenspace update method by incremental kernel PCA for realtime surveillance. Experimental results indicate that accuracy of proposed method is comparable to batch KPCA and outperforms than APEX. Furthermore proposed method has efficiency in memory requirement compared to KPCA.
\end{abstract}

\section{Introduction}

Unsupervised surveillance gadgets aided by hi-tech visual information retrieval and indexing systems use computerized face recognition techniques that can recognizes faces from an image. There are two main approaches for face recognition[1]. The first approach is the feature based matching approach using the relationship between facial features[2]. The second approach is the template matching approach using the holistic features of the face images[2]. Template based techniques often follow the subspace method called eigenface originated by Turk and Pentland[3]. This technique is based on the Karhunen-Loeve transformation, which is also referred as PCA. It has gained great success and become a de facto standard and a common performance benchmark in face recognition. One of the attractive characteristics of PCA is that a high demension vector can be represented by a small number of orthogonal basis vectors. The conventional methods

\footnotetext{
* This study was supported by a grant of the Korea Health 21 R\&D Project, Ministry of Health \& Welfare, Republic of Korea (A05-0909-A80405-05N1-00000A).
} 
of PCA such as singular value decomposion(SVD) and eigen-decomposition, perform in batch-mode with a computational complexity of $O\left(\mathrm{~m}^{3}\right)$ when $m$ is the minimum value between the data dimension and the number of training examples. Undoubtedly these methods are computationally expensive when dealing with large scale problems where both the dimension and the number of training examples are large. To address this problem, many researchers have been working on incremental algorithms. Among them Chandrasekaran et al presented an incremental eigenspace update method using SVD[4]. Hall et al derived an eigen-decomposition based incremental algorithm and later extended their work to merge and split eigenspace models[5]. Another problem of PCA is that it only defines a linear projection of the data, the scope of its application is necessarily somewhat limited. It has been shown that most of the data in the real world are inherently non-symmetric and therefore contain higher-order correlation information that could be useful[6]. PCA is incapable of representing such data. For such cases, nonlinear transforms is necessary. Recently kernel trick has been applied to PCA and is based on a formulation of PCA in terms of the dot product matrix instead of the covariance matrix[7]. Kernel PCA(KPCA), however, requires storing and finding the eigenvectors of a $N \times N$ kernel matrix where $N$ is a number of patterns. It is infeasible method for when $N$ is large. This fact has motivated the development of incremental way of KPCA method which does not store the kernel matrix. In this paper we propose a method that allows for incremental eigenspace update method by incremental kernel PCA for vision learning and recognition. Paper is organized as follows. In Section 2 we will briefly explain the incremental PCA method. In Section 3 KPCA is introduced and to make KPCA incrementally, empirical kernel map method is explained. Experimental results to evaluate the performance of proposed method is shown in Section 4. Discussion of proposed method and future work is described in Section 5 .

\section{Incremental PCA}

In this section, we will give a brief introduction to the method of incremental PCA algorithm which overcomes the computational complexity of standard PCA. Before continuing, a note on notation is in order. Vectors are columns, and the size of a vector, or matrix, where it is important, is denoted with subscripts. Particular column vectors within a matrix are denoted with a superscript, while a superscript on a vector denotes a particular observation from a set of observations, so we treat observations as column vectors of a matrix. As an example, $A_{m n}^{i}$ is the $i$ th column vector in an $m \times n$ matrix. We denote a column extension to a matrix using square brackets. Thus $\left[A_{m n} b\right]$ is an $(m \times(n+1))$ matrix, with vector $b$ appended to $A_{m n}$ as a last column.

To explain the incremental PCA, we assume that we have already built a set of eigenvectors $U=\left[u_{j}\right], j=1, \cdots, k$ after having trained the input images $\mathbf{x}_{i}, i=1, \cdots, N$. The corresponding eigenvalues are $\Lambda$ and $\overline{\mathbf{x}}$ is the mean of input image. Incremental building of eigenspace requires to update these eigenspace 
to take into account of a new input image. Here we give a brief summarization of the method which is described in [5]. First, we update the mean:

$$
\bar{x}^{\prime}=\frac{1}{N+1}\left(N \bar{x}+x_{N+1}\right)
$$

We then update the set of eigenvectors to reflect the new input image and to apply a rotational transformation to $U$. For doing this, it is necessary to compute the orthogonal residual vector $\hat{h}=\left(U a_{N+1}+\bar{x}\right)-x_{N+1}$ where $a_{N+1}$ is principal component and normalize it to obtain $h_{N+1}=\frac{h_{N+1}}{\left\|h_{N+1}\right\|_{2}}$ for $\left\|h_{N+1}\right\|_{2}>0$ and $h_{N+1}=0$ otherwise. We obtain the new matrix of eigenvectors $U^{\prime}$ by appending $h_{N+1}$ to the eigenvectors $U$ and rotating them :

$$
U^{\prime}=\left[U, h_{N+1}\right] R
$$

where $R \in \mathbf{R}_{(\mathbf{k}+\mathbf{1}) \times(\mathbf{k}+\mathbf{1})}$ is a rotation matrix. $R$ is the solution of the eigenspace of the following form:

$$
D R=R \Lambda^{\prime}
$$

where $\Lambda^{\prime}$ is a diagonal matrix of new eigenvalues. We compose $D \in \mathbf{R}_{(\mathbf{k}+\mathbf{1}) \times(\mathbf{k}+\mathbf{1})}$ as:

$$
D=\frac{N}{N+1}\left[\begin{array}{cc}
\Lambda & 0 \\
0^{T} & 0
\end{array}\right]+\frac{N}{(N+1)^{2}}\left[\begin{array}{cc}
a a^{T} & \gamma a \\
\gamma a^{T} & \gamma^{2}
\end{array}\right]
$$

where $\gamma=h_{N+1}^{T}\left(x_{N+1}-\bar{x}\right)$ and $a=U^{T}\left(x_{N+1}-\bar{x}\right)$. Though there are other ways to construct matrix $D[4][5]$, the only method ,however, described in [6] allows for the updating of mean.

\subsection{Updating Image Representations}

The incrementl PCA represents the input image with principal components $a_{i(N)}$ and it can be approximated as follows:

$$
\widehat{x}_{i(N)}=U a_{i(N)}+\bar{x}
$$

To update the principal components $a_{i(N)}$ for a new image $x_{N+1}$, computing an auxiliary vector $\eta$ is necessary. $\eta$ is calculated as follows:

$$
\eta=\left[U \widehat{h}_{N+1}\right]^{T}\left(\bar{x}-\bar{x}^{\prime}\right)
$$

then the computation of all principal components is

$$
a_{i(N+1)}=\left(R^{\prime}\right)^{T}\left[\begin{array}{c}
a_{i(N)} \\
0
\end{array}\right]+\eta, \quad i=1, \cdots, N+1
$$

The transformations described above yield a model that represents the input images with the same accuracy as the previous one, therefore we can now discard the old subspace and the coefficients that represent the image in it. $x_{N+1}$ is represented accurately as well, so we can safely discard it. The representation 
of all $N+1$ images is possible because the subspace is spanned by $k+1$ eigenvector. Due to the increase of the dimensionality by one, however, more storage is required to represent the data. If we try to keep a $k$-dimensional eigenspace, we lose a certain amount of information. In order to balance the storage requirements with the level of accuracy, it is needed for us to set the criterion on retaining the number of eigenvectors. There is no explicit guideline for retaining a number of eigenvectors.

In this paper we set our criterion on adding an eigenvector as $\lambda_{k+1}^{\prime}>0.7 \bar{\lambda}$ where $\bar{\lambda}$ is a mean of the $\lambda$. Based on this rule, we decide whether adding $u_{k+1}^{\prime}$ or not.

\section{Incremental KPCA}

A prerequisite of the incremental eigenspace update method is that it has to be applied on the data set. Furthermore incremental PCA builds the subspace of eigenvectors incrementally, it is restricted to apply the linear data. But in the case of KPCA this data set $\Phi\left(x^{N}\right)$ is high dimensional and most of the time can not even be calculated explicitly. For the case of nonlinear data set, applying feature mapping function method to incremental PCA may be one of the solutions. This is performed by so-called kernel-trick, which means an implicit embedding to an infinite dimensional Hilbert space[9](i.e. feature space) $F$.

$$
K(x, y)=\Phi(x) \cdot \Phi(y)
$$

Where $K$ is a given kernel function in an input space. When $K$ is semi positive definite, the existence of $\Phi$ is proven[7]. Most of the case ,however, the mapping $\Phi$ is high-dimensional and cannot be obtained explicitly. The vector in the feature space is not observable and only the inner product between vectors can be observed via a kernel function. However, for a given data set, it is possible to approximate $\Phi$ by empirical kernel map proposed by Scholkopf[10] and Tsuda[11] which is defined as $\Psi_{N}: \mathbf{R}^{d} \rightarrow \mathbf{R}^{N}$

$$
\begin{aligned}
\Psi_{N}(x) & =\left[\Phi\left(x_{1}\right) \cdot \Phi(x), \cdots, \Phi\left(x_{N}\right) \cdot \Phi(x)\right]^{T} \\
& =\left[K\left(x_{1}, x\right), \cdots, K\left(x_{N}, x\right)\right]^{T}
\end{aligned}
$$

A performance evaluation of empirical kernel map was shown by Tsuda. He shows that support vector machine with an empirical kernel map is identical with the conventional kernel map[12]. The empirical kernel map $\Psi_{N}\left(x_{N}\right)$, however, do not form an orthonormal basis in $\mathbf{R}^{N}$, the dot product in this space is not the ordinary dot product. In the case of KPCA, however, we can be ignored as the following argument. The idea is that we have to perform linear PCA on the $\Psi_{N}\left(x_{N}\right)$ from the empirical kernel map and thus diagonalize its covariance matrix. Let the $N \times N$ matrix $\Psi=\left[\Psi_{N}\left(x_{1}\right), \Psi_{N}\left(x_{2}\right), \ldots, \Psi_{N}\left(x_{N}\right)\right]$, then from equation (9) and definition of the kernel matrix we can construct $\Psi=N K$. The covariance matrix of the empirically mapped data is:

$$
C_{\Psi}=\frac{1}{N} \Psi \Psi^{T}=N K K^{T}=N K^{2}
$$


In case of empirical kernel map, we diagonalize $N K^{2}$ instead of $K$ as in KPCA. Mika shows that the two matrices have the same eigenvectors $\left\{u_{k}\right\}[12]$. The eigenvalues $\left\{\lambda_{k}\right\}$ of $K$ are related to the eigenvalues $\left\{k_{k}\right\}$ of $N K^{2}$ by

$$
\lambda_{k}=\sqrt{\frac{k_{k}}{N}}
$$

and as before we can normalize the eigenvectors $\left\{v_{k}\right\}$ for the covariance matrix $C_{\Psi}$ of the data by dividing each $\left\{u_{k}\right\}$ by $\sqrt{\lambda_{k} N}$. Instead of actually diagonalize the covariance matrix $C_{\Psi}$, the incremental KPCA is applied directly on the mapped data $\Psi=N K$. This makes it easy for us to adapt the incremental eigenspace update method to KPCA such that it is also correctly takes into account the centering of the mapped data in an incremental way. By this result, we only need to apply the empirical map to one data point at a time and do not need to store the $N \times N$ kernel matrix.

\section{Experiment}

To evaluate the performance of accuracy on eiegnspace update for incremental data we take nonlinear data. The disadvantage of incremental method is their accuracy compared to batch method even though it has the advantage of memory efficiency. So we shall apply proposed method to a simple toy data which will show the accuracy and memory efficiency of incremental KPCA compared to APEX model proposed by Kung[13] and batch KPCA. Next we will use images from the Columbia Object Image Library(COIL-20). The set is consisted of images of 20 objects rotated about their vertical axis, resulting in 72 images per objects. We used these images for testing the performance of incremental KPCA.

\subsection{Toy Data}

To evaluate the eigenspace update accuracy and memory efficiency of incremental KPCA compared to APEX and KPCA we take nonlinear data used by Scholkoff[8]. Totally 41 training data set is generated by:

$$
y=x^{2}+0.2 \varepsilon: \varepsilon \text { from } N(0,1), x=[-1,1]
$$

First we compare feature extraction ability of incremental KPCA to APEX model. APEX model is famous principal component extractor based on Hebbian learning rule. Applying toy data to incremental KPCA we finally obtain 2 eigenvectors. To evaluate the performance of two methods on same condition, we set 2 output nodes to standard APEX model.

In table 1 we experimented APEX method on various conditions. Generally neural network based learning model has difficulty in determining the parameters; for example learning rate, initial weight value and optimal hidden layer node. This makes us to conduct experiments on various conditions. $\|w\|$ is norm of weight vector in APEX and $\|w\|=1$ means that it converges stable minimum. $\cos \theta$ is angle between eigenvector of KPCA and APEX, incremental 
Table 1. Performance evaluation of incremental KPCA(IKPCA) and APEX

\begin{tabular}{|c|c|c|c|c|c|c|c|}
\hline Method & Iteration & Learning Rate & $w_{1}$ & $w_{2}$ & $\cos \theta_{1}$ & $\cos \theta_{2}$ & MSE \\
\hline APEX & 50 & 0.01 & 0.6827 & 1.4346 & 0.9993 & 0.7084 & 14.8589 \\
\hline APEX & 50 & 0.05 & & & & do not converge & \\
\hline APEX & 500 & 0.01 & 1.0068 & 1.0014 & 0.9995 & 0.9970 & 4.4403 \\
\hline APEX & 500 & 0.05 & 1.0152 & 1.0470 & 0.9861 & 0.9432 & 4.6340 \\
\hline APEX & 1000 & 0.01 & 1.0068 & 1.0014 & 0.9995 & 0.9970 & 4.4403 \\
\hline APEX & 1000 & 0.05 & 1.0152 & 1.0470 & 0.9861 & 0.9432 & 4.6340 \\
\hline IKPCA & 100 & & 1 & 1 & 1 & 1 & 0.0223 \\
\hline
\end{tabular}

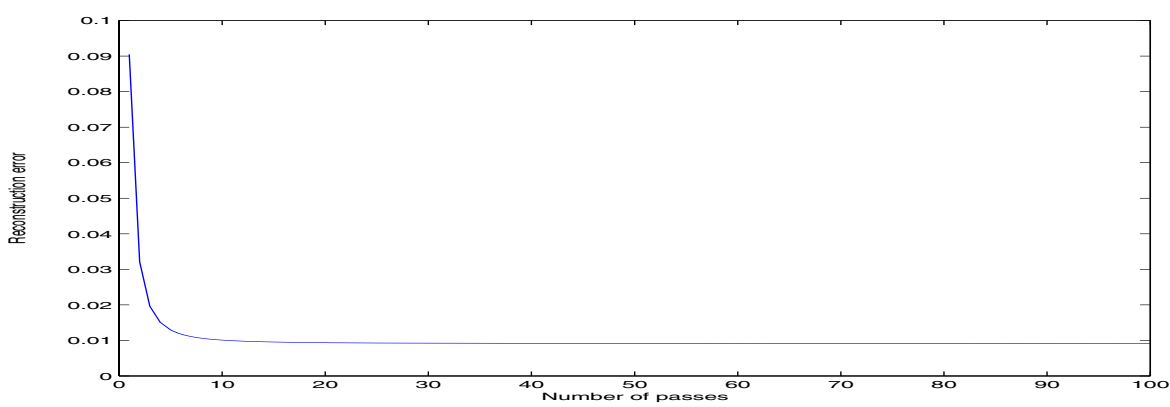

Fig. 1. Reconstruction error change by re-learning in incremental KPCA

KPCA respectively. $\cos \theta$ of eigenvector can be a factor of evaluating accuracy how much incremental KPCA and APEX is close to accuracy of KPCA. Table 1 nicely shows the two advantages of incremental KPCA compared to APEX: first, performance of incremental KPCA is better than APEX; second, the performance of incremental KPCA is easily improved by re-learning. Another factor of evaluating accuracy is reconstruction error. Reconstruction error is defined as the squared distance between the $\Psi$ image of $x_{N}$ and reconstruction when projected onto the first $i$ principal components.

$$
\delta=\left|\Psi\left(x_{N}\right)-P_{l} \Psi\left(x_{N}\right)\right|^{2}
$$

In here $P_{l}$ is the first $i$ principal component. The MSE(Mean Square Error) value of reconstruction error in APEX is 4.4403 whereas incremental KPCA is 0.0223 . This means that the accuracy of incremental KPCA is superior to standard APEX and similar to that of batch KPCA. Figure 1 shows the MSE value change for reconstruction error by re-learning in incremental KPCA. Relearning is similar meaning of epoch in neural network learning. We can see that the performance of incremental KPCA is easily improved by re-learning. Above results of simple toy problem indicate that incremental KPCA is comparable to the batch way KPCA and superior in terms of accuracy. 
Table 2. Memory efficiency of incremental KPCA compared to KPCA on toy data

\begin{tabular}{|c|c|c|}
\hline & KPCA & IKPCA \\
\hline Kernel matrix & 41 X 41 & none \\
\hline R matrix & none & 3 X 3 \\
\hline D matrix & none & 3 X 3 \\
\hline Efficiency ratio & 93.3889 & 1 \\
\hline
\end{tabular}

Next we will compare the memory efficiency of incremental KPCA compared to $\mathrm{KPCA}$. In this experiments, incremental KPCA only needs D matrix and R matrix whereas KPCA needs kernel matrix. Table 2 shows the memory requirement of each method. Memory requirement of standard KPCA is 93 times more than incremental KPCA. We can see that incremental KPCA is more efficient in memory requirement than KPCA and has similar ability of eigenspace update accuracy. By this simple toy problem we can show that incremental KPCA has similar accuracy compare to KPCA and more efficient in memory requirement than KPCA.

\subsection{Reconstruction Ability}

To compare the reconstruction ability of incremental eigenspace update method proposed by Hall to APEX model we conducted experiment on face data. Applying this data to incremental eigenspace update method we finally obtain 30 Eigenvectors. As earlier experiment we set 30 output nodes to standard APEX

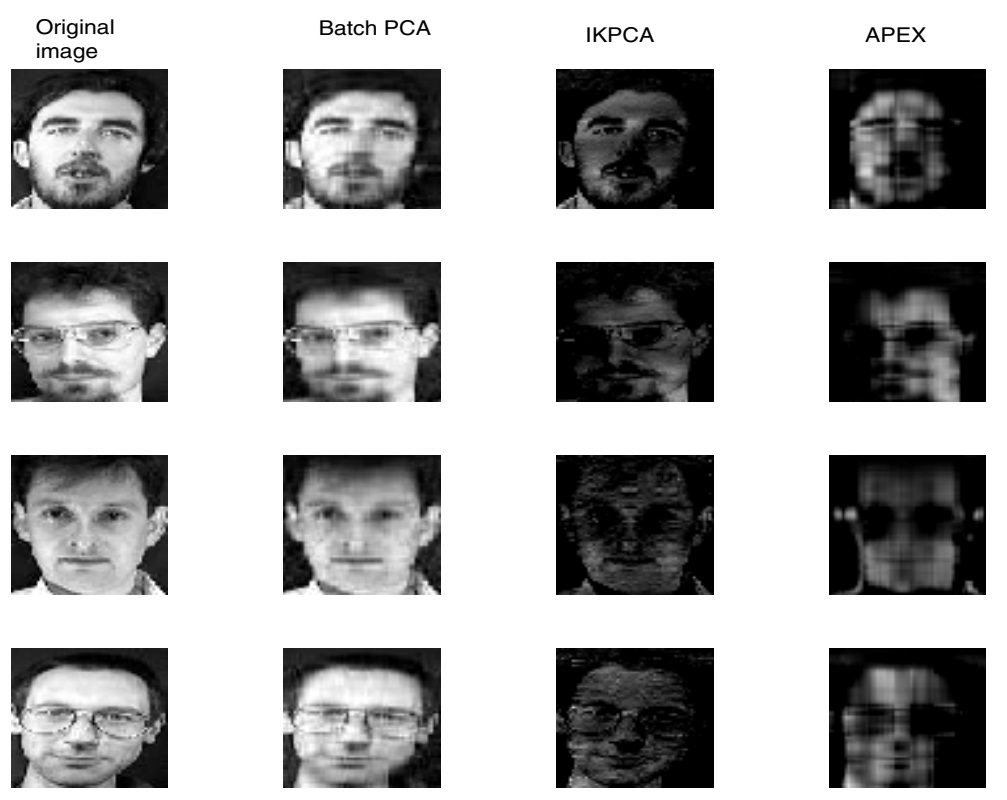

Fig. 2. Reconstructed image by incremental KPCA, APEX and batch PCA 
method. Figure 2 shows the original data and their reconstructed images by incremental KPCA method, batch PCA and APEX respectively. The MSE(Mean Square Error) value of reconstruction error in APEX is 10.159 whereas incremental KPCA is 0.26941 and KPCA is 0.15762 . This means that the accuracy of incremental KPCA is superior to standard APEX and similar to that of batch KPCA. We can see that reconstructed images by incremental KPCA update method is similar to original image and more clear compared to APEX method.

\section{Conclusion and Remarks}

A real time feature extraction for realtime surveillance system is proposed in this paper. We use incremental KPCA method in order to represent images in a low-dimensional subspace for realtime surveillance. Proposed method allows discarding the acquired images immediately after the update. By experimental results we can show that incremental KPCA has similar accuracy compare to KPCA and more efficient in memory requirement than KPCA. This makes proposed model is suitable for real time surveillance system. We will extend our research to realtime face recognition based on this research.

\section{References}

1. Chellappa, R. Wilson, C.L and Sirohey, S.: Human and machine recognition of faces:a survey Proc. of IEEE, vol.83, N0.5, May (1995) 705-740

2. Brunelli, R. and Poggio, T.:Face recognition:feature versus templates. IEEE Trans. PAMI, vol. 15, no. 10, (1993) 1042-1052

3. Turk, M. and Pentland, A.:Face recognition using eigenfaces. Proc. IEEE Conf. on CVPR, (1991) 586-591

4. Winkeler, J. Manjunath, B.S. and Chandrasekaran, S.:Subset selection for active object recognition. In CVPR, volume 2, IEEE Computer Society Press, June (1999) 511-516

5. Hall, P. Marshall, D. and Martin, R.: On-line eigenalysis for classification. In British Machine Vision Conference, volume 1, September (1998) 286-295

6. Softky, W.S and Kammen, D.M.: Correlation in high dimensional or asymmetric data set: Hebbian neuronal processing. Neural Networks vol. 4, Nov. (1991) 337-348

7. Gupta, H., Agrawal, A.K., Pruthi, T., Shekhar, C., and Chellappa., R.:An Experimental Evaluation of Linear and Kernel-Based Methods for Face Recognition. accessible at http://citeseer.nj.nec.com.

8. Murakami, H. Kumar.,B.V.K.V.:Efficient calculation of primary images from a set of images. IEEE PAMI, 4(5) (1982) 511-515

9. Vapnik, V. N.:Statistical learning theory. John Wiley \& Sons, New York (1998)

10. Scholkopf, B. Smola, A. and Muller, K.R.:Nonlinear component analysis as a kernel eigenvalue problem. Neural Computation 10(5), (1998) 1299-1319

11. Tsuda, K.:Support vector classifier based on asymmetric kernel function. Proc. ESANN (1999)

12. Mika, S.:Kernel algorithms for nonlinear signal processing in feature spaces. Master's thesis, Technical University of Berlin, November (1998)

13. Diamantaras, K.I. and Kung, S.Y.:Principal Component Neural Networks: Theory and Applications. New York John Wiley \& Sons, Inc (1996) 